\title{
Accuracy Analysis of a New Reference-free Digital Temperature Sensor in CMOS
}

\author{
Sami Ur Rehman \\ Department of Electrical Engineering and Computer Sciences \\ Masdar Institute of Science and Technology, Abu Dhabi, United Arab Emirates \\ srehman@masdar.ac.ae
}

\begin{abstract}
A CMOS digital temperature measurement technique is reported, which doesn't require an explicit reference voltage during temperature to digital conversion. Instead, one of the inputs from the sensor core serves as the reference. After eliminating the need to use an external reference, we observe at least twofold increase in temperature estimation accuracy compared to the reference based traditional approach. Designed in $65 \mathrm{~nm}$ CMOS, the digital sensor consume a total power of $220 \mu \mathrm{V}$ and an area of $0.25 \mathrm{~mm}^{2}$, and achieves a $\pm 3 \sigma$ accuracy of $1^{\circ} \mathrm{C}$ after curvature correction and room temperature trim.
\end{abstract}

Keywords- reference-free, analog to digital conversion, thermal sensor core, accuracy.

\section{INTRODUCTION}

Reference circuits are considered an essential part of any digital sensor front-end circuit. Since the reference is a value against which the input is compared, the digitization of a time-varying input in a sensor readout system typically requires a PVT immune and stable reference. A Bandgap Reference (BGR) circuit introduced first in [1] is still a popular choice of implementation for many applications. The idea is based on adding a quantity complementary to temperature (CTAT) like $\mathrm{V}_{\mathrm{BE}}$ or $\mathrm{V}_{\mathrm{GS}}$ to a quantity proportional to temperature (PTAT) like $\Delta \mathrm{V}_{\mathrm{BE}}$ or $\Delta \mathrm{V}_{\mathrm{GS}}$ such that their temperature-dependent slopes cancel out, resulting in a temperature independent BGR $\left(\mathrm{V}_{\mathrm{BGR}}=\mathrm{V}_{\mathrm{BE}}+\alpha \Delta \mathrm{V}_{\mathrm{BE}}\right.$, where $\alpha$ is a design dependent multiplication factor). Looking closely, we observe that a BGR circuit is achieved through a combination of three arithmetic operations .i.e. subtraction, multiplication, and addition. Analog implementation of these operations introduces nonlinearity and error in the reference voltage, making it a sensitive circuit. Some of the common error sources which can potentially corrupt the output of BJT based BGR are 1) Op-amp Offset: PTAT current generation circuit requires an op-amp to generate difference of the emitter base voltage. Offset associated with this op-amp could be as large as $\pm 10 \mathrm{mV}$ and can contribute largest error to BGR circuit , 2) Process Variations in $V_{B E}$ : Since $\mathrm{V}_{\mathrm{BE}}=k T / q \times \ln \left(\mathrm{I}_{\mathrm{C}} / \mathrm{I}_{\mathrm{S}}\right)$, process variations can result from the variations in saturation current $\left(\mathrm{I}_{\mathrm{S}}\right)$ or collector current $\left(\mathrm{I}_{\mathrm{C}}\right)$. Spread in $\mathrm{I}_{\mathrm{S}}$ could be due to variations in base doping and transistor dimensions. Is can change by as much as $40 \%$ of its nominal value in even a mature CMOS node [2], 3) Process Variations in $\Delta V_{B E}: \Delta \mathrm{V}_{\mathrm{BE}}$ is generated by forcing currents with different current densities in the BJTs. Temperature drift in the ratio of these currents can impact the accuracy of the BGR. 4) Process Spread and Mismatch in Bias Resistor: Process spread and mismatch in the bias resistors used to generate the PTAT current can result in a PTAT error, 4) Emitter-Base Series Resistance: Typical values of emitter-base series resistance in parasitic vertical pnp could be as much as 250 ohms. Voltage drop across this resistance adds error to $\left.\mathrm{V}_{\mathrm{EB}}, 5\right)$ B BR Curvature: In reality, $V_{\mathrm{BE}}$ does not strictly posses first order temperature coefficient and is actually slightly nonlinear over a wide temperature range. This curvature of $\mathrm{V}_{\mathrm{EB}}$ is carried forward to the BGR, and limits its precision.

However, since [1], many variants of BGR have been reported, some with military-grade precision. But, such accuracy can only be achieved through precision-enhancement and linearity-compensation techniques. Mixed-signal implementation of such error correction circuits in scaled CMOS nodes is challenging due to presence of various short channel effects. Also, a penalty in terms of power and area consumption has to be paid. Therefore, current trend is to push these error correction circuits into digital domain or, even better, to resort to techniques capable of moving the arithmetic operations of BGR itself into the digital world.

In the realm of temperature sensor design, one such technique was pioneered by Makinwa in his numerous publications .e.g. [3]. In [3], we observe that two of the three arithmetic operations were implicitly implemented in the digital domain; however, the difference operation is still being performed in analog domain through a difference amplifier. Now, we present a smart sensor with all arithmetics of BGR pushed into the digital backend. The theoretical concept of the reference-free scheme was first proposed in our prior publication [4]; however, this paper provides in detail the accuracy analysis of measured results.

\section{THEORETICAL BACKGROUND}

When two BJT devices are operated at different current densities, the difference of their base-emitter voltages is directly proportional to absolute temperature $\left(\Delta \mathrm{V}_{\mathrm{BE}}=\mathrm{V}_{\mathrm{BE} 2}-\mathrm{V}_{\mathrm{BE} 1}=k T / q \times \ln (n)\right)[1]$, where 
$n$ represents difference of current densities, as can be seen in Fig 1. This relationship has been exploited to design precise thermal sensors in silicon. It is also clear from [1] that the outputs of the thermal sensor, $\mathrm{V}_{\mathrm{BE} 1}$ and $\mathrm{V}_{\mathrm{BE} 2}$, can be utilized to implement the BGR circuit. This BGR reference, along with an Analog-toDigital Converter (ADC), digitizes one of the two outputs of the thermal sensor or their difference. The resulting $\mathrm{ADC}$ ratio of input over reference can be interpreted in a way such that one of the inputs from the sensor core serves as the reference. In other words, since a BGR is an arithmetic combination of outputs from the sensor core, we can use one input as a reference to the $\mathrm{ADC}$ and can implement the remaining arithmetic operations needed for a BGR after the ADC in the digital backend. To theoretically manifest this qualitative interpretation of referencefree digital temperature sensor we note that the output of any ADC is the ratio of input to the reference. This means the ADC output, $\mu$, for the thermal sensor can be written as:

$$
\begin{gathered}
\mu=\frac{V_{I N}}{V_{R E F}}=\frac{\alpha \times \Delta V_{B E}}{V_{B E}+\left(\alpha \times \Delta V_{B E}\right)} \\
\mu=\frac{V_{I N}}{V_{R E F}}=\frac{1}{1+\frac{V_{B E}}{\alpha \Delta V_{B E}}} \\
\mu=\frac{V_{I N}}{V_{R E F}}=\frac{1}{1+\frac{1}{\alpha\left(V_{B E 2} / V_{B E 1}-1\right)}}
\end{gathered}
$$

Eq (1) shows theoretically similar but practically different digitization schemes for the traditional, Makinwa [3], and the proposed method in Eq 1a, 1b and $1 \mathrm{c}$ respectively. Traditional scheme uses external voltage reference while implementing all three arithmetic operations of BGR in analog domain as shown in Fig 1a, [3] uses $\Delta \mathrm{V}_{\mathrm{BE}}$ as reference and $\mathrm{V}_{\mathrm{BE}}$ as input ( $\mathrm{ADC}$ output is $\mathrm{X}=\mathrm{V}_{\mathrm{BE}} / \Delta \mathrm{V}_{\mathrm{BE}}$ ), pushing two of the three arithmetic operations into digital backend. The proposed scheme uses lower of the two $V_{B E}$ voltages as input while other as reference (ADC output is $\mathrm{X}=\mathrm{V}_{\mathrm{BE} 1} / \mathrm{V}_{\mathrm{BE} 2}$ ) as can be seen in Fig $1 \mathrm{~b}$. In [4], we proposed theoretical background of two reference-free successive approximation (SAR) ADC based smart thermal sensors. However, only the ratiometric approach, discussed above, has been implemented in silicon and its measured accuracy results are presented here.

\section{CIRCUIT IMPLEMENTATION}

\section{A. Sensor Core}

The sensor core consists of two parasitic vpnps biased with PTAT currents of different current densities (Since vpnps are diodes biased through emitters, we will refer to $\mathrm{V}_{\mathrm{BE}}$ as $\mathrm{V}_{\mathrm{EB}}$ ). Vertical parasitic vpnps available in CMOS have to be biased through emitter.
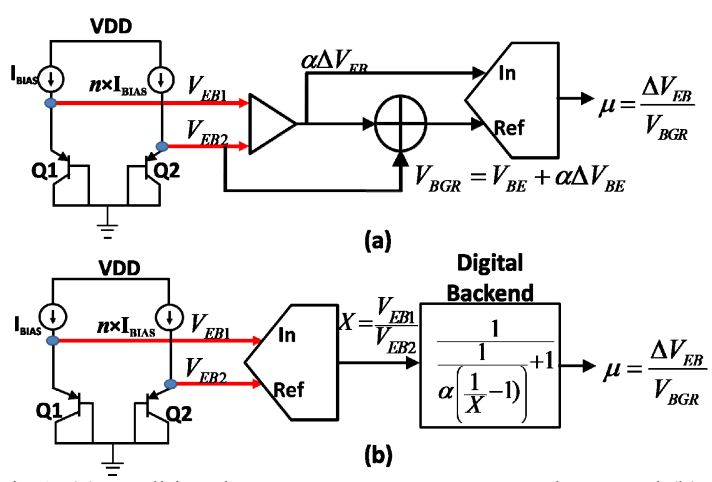

Fig 1. (a) Traditional temperature measurement scheme and (b) the proposed scheme.

This results in Vbe being dependent on the common emitter current gain, $\beta$. Variations in $\beta$ result in variations in Vbe and hence affects the accuracy of the sensor. The nominal value of $\beta$ is around 1 in $65 \mathrm{~nm}$ CMOS process as can be seen in Fig 2a.

To eliminate the dependency of $\beta$, Q1 and Q2 have to be biased with PTAT current. The circuit on the left side of Fig 2b generates PTAT current by forcing the nodes $\mathrm{X}$ and $\mathrm{Y}$ to have similar voltage using the positive feedback, and copies the generated PTAT current into temperature sensing BJTs, Q1 and Q2, using MOS current mirrors, M4 and M5. Dynamic element matching was implemented in the current mirrors; however, it is not shown in the Fig $2 b$ for simplicity. In another variant the positive feedback was replaced with an op-amp. Further details of this circuit could be found in [4].

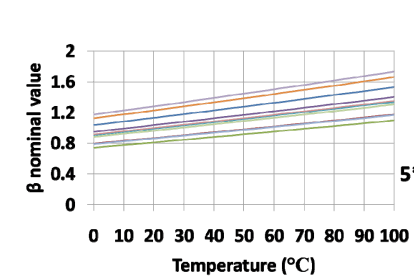

(a)

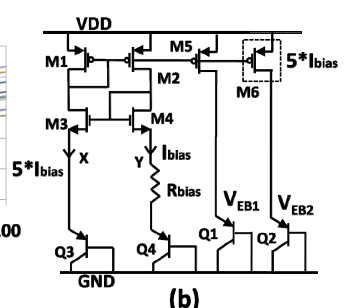

(b)
Fig 2. (a) Statistical nominal simulated values of $\beta$ in $65 \mathrm{~nm}$ CMOS, (b) Designed sensor core circuit with PTAT current generator

\section{B. 12-bit SAR ADC}

In the following stage, the implemented 12-bit data converter is split capacitor array fully differential Successive Approximation Register (SAR) ADC with unit capacitor of $20 \mathrm{fF}$. The ADC, shown in Fig. 3, is monotonically switched and follows set and up SAR scheme (after sampling input, all bottom plates are grounded and then connected to $V_{R E F}$, or remain grounded, as determined by SAR logic). $\mathrm{V}_{\mathrm{EB} 2}$ is first sampled on the top capacitor plate of both Digital-toAnalog (DAC) capacitive arrays. Meanwhile, the bottom plate of the DAC array is connected to $V_{\mathrm{EB} 1}$ and the bottom plate of bottom DAC array is connected to $\mathrm{V}_{\mathrm{EB} 2}$. Next the bottom plates of both DAC arrays are grounded. Afterwards regular SAR scheme is initiated and bottom plates of DAC arrays are set/reset depending on the output of the comparator. The comparator is composed of a 3-stage amplifier followed by a dynamic latch and consumes 


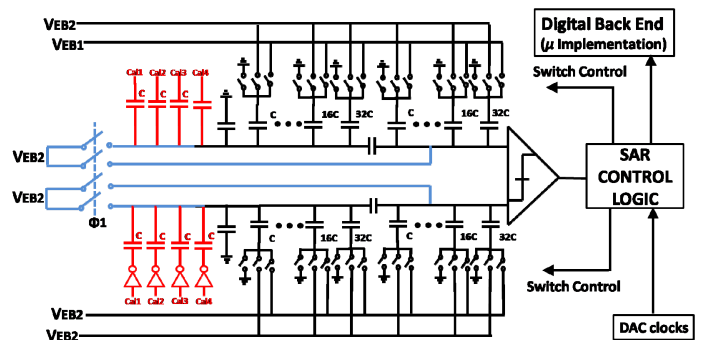

Fig 3. Designed 12-bit split capacitor SAR ADC with 4-bit calibration DAC and digital backend

$30 \mu \mathrm{A}$ of current. The SAR ADC yields $\mathrm{X}=\mathrm{V}_{\mathrm{EB} 1} / \mathrm{V}_{\mathrm{EB} 2}$ and the digital backend was implemented as a software estimation routine.

Redundancy provides tolerance to comparator decision errors and eliminates wide codes. We employ 4-bit DAC array to provide redundancy which can tolerate up to $4 \mathrm{LSBs}$ of comparator error. In SAR ADCs calibration is a process that measures and corrects for capacitor mismatch errors. Although we implemented calibration for errors in the largest 4 capacitors in our SAR architecture, the range of code values exercised by $\mathrm{X}$ is designed so the $3 \mathrm{MSB}$ capacitor mismatch contribution becomes a simple offset. This is due to the value of $\mathrm{X}$ being in between the major code transitions contributed by the $3 \mathrm{MSBs}$. Since the nonlinearity due to these capacitor errors becomes effectively an offset, it is eliminated using a simple room temperature trim. The fact that the 3 MSBs do not change over the temperature range of interest can be used to eliminate the first three SAR algorithm steps to save power and conversion time, but this was not implemented in this prototype. The calibration scheme sets the 4-bit calibration DAC dynamically during SAR operation.

\section{MEASUREMENT RESULTS AND COMPARISON}

Micrograph of the designed 65nm CMOS microchip and the measurement setup can be seen in Fig 4. The measurement setup included a thermal chamber, data acquisition IO card, reference RTD Pt-100 thermister and measurement software. The designed thermal sensor was placed inside the chamber along with reference thermister. The DUT PCB was connected to the software through the IO card. For temperature accuracy measurements the chamber temperature was

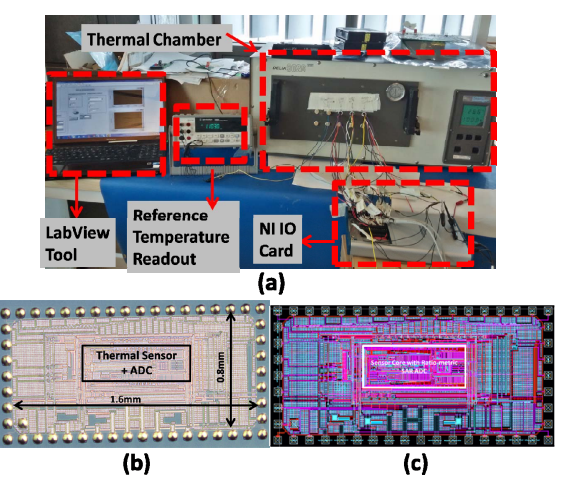

Fig 4. (a) Temperature measurement setup, (b) chip micrograph, and (c) chip layout swept from $25^{\circ} \mathrm{C}$ up to $100^{\circ} \mathrm{C}$.

To provide a comparison of the temperature estimates based on the ratio and difference of $\mathrm{V}_{\mathrm{EB}}$, we collected data for $\mathrm{V}_{\mathrm{EB}}$ versus temperature from 12 chips using a 16-bit acquisition card. To eliminate the contribution of random errors during measurements, for each chip at every temperature, 20000 samples were taken and its average value was used to report the error at that temperature.

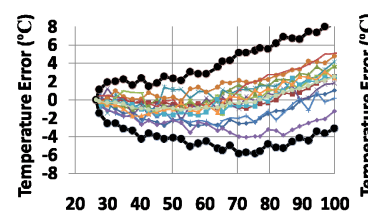

Temperature (
$\left({ }^{\circ} \mathrm{C}\right)$

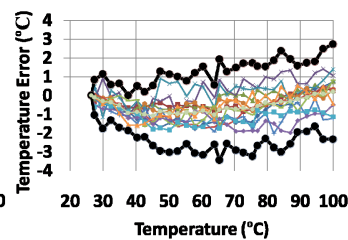

(b)
Fig 5. (a) Raw temperature estimation error from the sensor core using reference based approach and (b) reference-free approach with $\pm 3 \sigma$ limits and room temperature trim for 12 chip samples.

Fig 5 provides the statistical comparison of raw temperature sensor core error between the proposed and traditional temperature estimation schemes (BGR was estimated from the measured values of $V_{E B 1}$ and $\mathrm{V}_{\mathrm{EB} 2}$ ). Thick black lines in the figures represent $\pm 3 \sigma$ values of errors over a temperature ranging from $25^{\circ} \mathrm{C}$ to $100^{\circ} \mathrm{C}$ for 12 chip samples. Fig 5 clearly suggests using the proposed scheme results in two times better accuracy compared to traditional implementation.

Process alterations in parameters like saturation current, collector current, bias resistors, base-emitter resistance etc can result in base-emitter voltage changing the polarity of its TC over temperature; usually called BJT curvature. This curvature is visibly seen in Fig 5. We computed curvature correction equations using technique presented in [5] for all chip samples over the given temperature range. For the proposed scheme we achieved correction coefficients

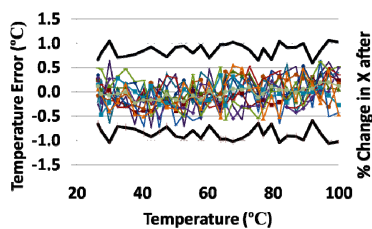

a)

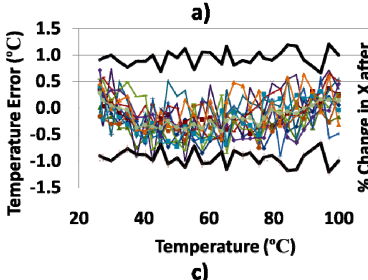

c)

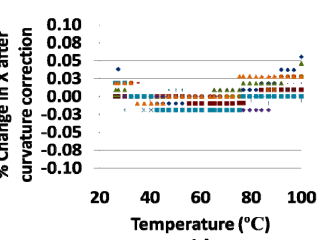

Temperature $\left({ }^{\circ} \mathrm{C}\right)$

b)

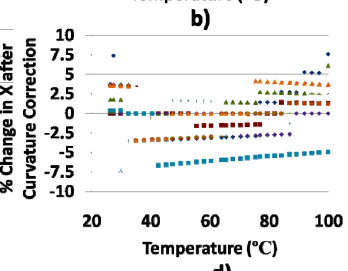

d)
Fig 6. (a) Curvature corrected temperature error for proposed approach with $\pm 3 \sigma$ limits and (b) the corresponding \% change in the value of $\mathrm{V}_{\mathrm{EB} 1} / \mathrm{V}_{\mathrm{EB} 2}$. (c) Curvature corrected temperature error for the traditional approach with $\pm 3 \sigma$ limits and (d) the corresponding $\%$ change in the value of $\mathrm{X}\left(\Delta \mathrm{V}_{\mathrm{EB}} / \mathrm{V}_{\mathrm{REF}}\right)$.

which changed the value of $\mathrm{V}_{\mathrm{EB} 1} / \mathrm{V}_{\mathrm{EB} 2}$ by a maximum of $\pm 0.05 \%$ from its nominal value across the entire temperature range as can be seen in Fig $6(\mathrm{a} \& \mathrm{~b})$. Curvature correction resulted in $\pm 3 \sigma$ accuracy of within $1^{\circ} \mathrm{C}$. To achieve the same accuracy in traditional approach we had to adjust curvature 
coefficients in correction equations which changed the value of $\Delta \mathrm{V}_{\mathrm{EB}} / \mathrm{V}_{\mathrm{REF}}$ by as much as $\pm 7.5 \%$ of its nominal value compared to mere $\pm 0.05 \%$ in proposed scheme, shown in Fig 6(c\&d). Bringing $\pm 7.5 \%$ change, compared to $\pm 0.05 \%$, requires considerably higher power and area overhead when these equations are implemented in hardware.

The outputs of the sensor core, $\mathrm{V}_{\mathrm{EB} 1}$ and $\mathrm{V}_{\mathrm{EB} 2}$, were directly applied to the input and reference terminals of the on-chip 12-bit SAR ADC. Fig 7a shows the error resulting at the output of the ADC for 6 chip samples. Fig $7 b$ shows the Differential Non-linearity (DNL) plot of the SAR ADC. A fine ramp signal was applied at the input terminal of the $\mathrm{ADC}$ and varied from $0.5 \mathrm{~V}$ to $1.1 \mathrm{~V}$. This input was generated using the IO board with 16-bit voltage step resolution. To avoid the noise issues on the internal $\mathrm{V}_{\mathrm{BE} 2}$ reference to the $\mathrm{ADC}$, an external1.1V was applied to the reference input.

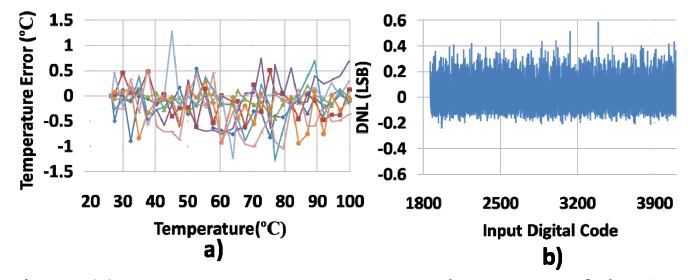

Fig 7. (a) Raw temperature error at the output of the ADC with room temperature trim for 6 chip samples. (b) DNL of the designed 12-bit SAR ADC

Fig 8 (a\&b) shows error estimated following the traditional approach of using a BGR circuit as reference and $\Delta \mathrm{V}_{\mathrm{EB}}$ as input with a room temperature trim and two point trim respectively. Fig 8 (c\&d) shows error results of sensor core from our proposed temperature estimation methodology with $\mathrm{V}_{\mathrm{EB} 1}$ as input and $\mathrm{V}_{\mathrm{EB} 2}$ as reference. Fig 8 (e\&f) presents error result after curvature correction and Fig 8 (g\&f) shows the estimated error at the output of the ADC.

Table I compares the specifications of the designed sensor with state of the art thermal sensors in submicron CMOS nodes which utilize similar sensing mechanism as ours mechanism as ours.

TABLE I

Comparison of Designed SENSOR With State of the ART

\begin{tabular}{|c|c|c|c|c|c|}
\hline Sensor & $\begin{array}{c}\text { Sense } \\
\text { Mech. }\end{array}$ & $\begin{array}{c}\text { Inaccuracy } \\
\left( \pm^{\circ} \mathrm{C}\right)\end{array}$ & $\begin{array}{c}\text { Process } \\
\text { Node } \\
(\mathrm{nm})\end{array}$ & $\begin{array}{c}\text { Temp } \\
\text { Range } \\
\left({ }^{\circ} \mathrm{C}\right)\end{array}$ & $\begin{array}{c}\text { Trim } \\
\text { Points }\end{array}$ \\
\hline$[6]$ & $\mathrm{V}_{\mathrm{BE}} / \mathbf{V}_{\mathrm{REF}}$ & 1.5 & 14 & $\mathbf{0}$ to 100 & 2 \\
\hline$[7]$ & $\mathrm{I}_{\mathrm{GS} 1} / \mathbf{I}_{\mathrm{GS} 2}$ & 1.6 & 65 & $\mathbf{0}$ to 100 & 2 \\
\hline$[8]$ & $\mathrm{V}_{\mathrm{BE}} / \mathbf{V}_{\mathrm{REF}}$ & 1.5 & 22 & -10 to & 2 \\
\hline $\begin{array}{c}\text { This } \\
\text { Work }\end{array}$ & $\mathrm{V}_{\mathrm{EB} 1} / \mathbf{V}_{\mathrm{EB} 2}$ & 1 & 65 & $\mathbf{2 5}$ to 100 & 1 \\
\hline
\end{tabular}

\section{CONCLUSION}

A new reference-free digital temperature estimation scheme is presented which provides $\pm 1^{\circ} \mathrm{C} 3 \sigma$ error inaccuracy with only room temperature trim in $65 \mathrm{~nm}$ CMOS. Comparison to state of the art proves the

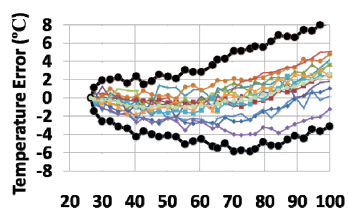

$20 \quad 3040 \quad 5060 \quad 70 \quad 8090100$ Temperature $\left({ }^{\circ} \mathrm{C}\right)$
(a)

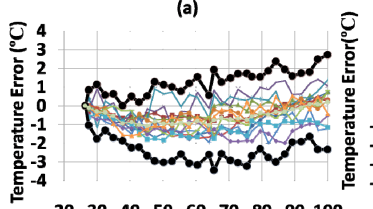

$\begin{array}{lllllll}30 & 40 \quad 60 & 70 \quad 80 & 90 & 100\end{array}$ Temperature $\left({ }^{\circ} \mathrm{C}\right)$

(c)

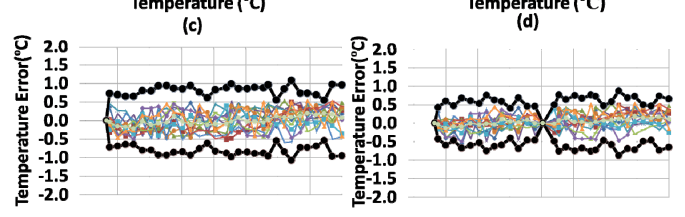

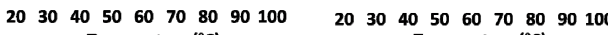
Temperature $\left({ }^{\circ} \mathrm{C}\right)$

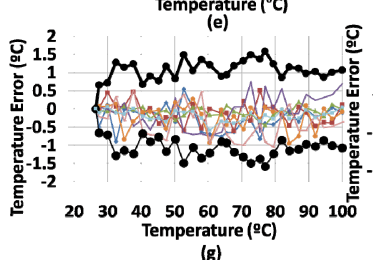

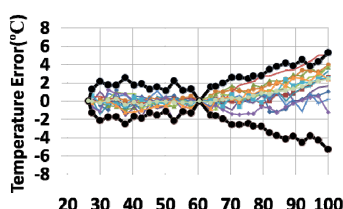

$\underset{(b)}{\text { Temperature }\left({ }^{\circ} \mathrm{C}\right)}$

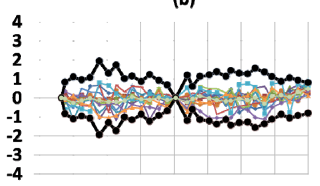

$40 \quad 50 \quad 60708090100$ (f)

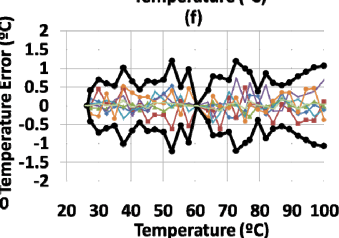

Fig 8. Statistical measured temperature error plots with $\pm 3 \circ$ limits. (a\&b) Sensor core temperature error for traditional approach, (c\&d) proposed approach, (e\&f) proposed approach with curvature correction from 12 chip samples.(g\&h) Sensor core with SAR ADC temperature error for the proposed approach from 6 chip samples.

proposed scheme provides accuracy advantage over the existing designs without employing any error compensation and linearity correction circuits.

\section{REFERENCES}

[1] D. F. Hilbiber, "A new semiconductor voltage standard," IEEE ISSCC Dig. Tech. Papers, pp. 32-33, 1964.

[2] V. G. Ceekala et al., "A method for reducing the effects of randommismatches in CMOS bandgap references," IEEE ISSCC Dig. Tech. Papers, pp. 318-319, 2002.

[3] K. Souri, Y. Chae, K.A.A Makinwa, "A CMOS temperature sensor with voltage-calibrated inaccuracy of $\pm 0.5^{\circ} \mathrm{C}(3 \sigma)$ from $55^{\circ} \mathrm{C}$ to $125^{\circ} \mathrm{C}$ ", IEEE J. Solid-State Circuits, vol. 48 , no. 1 , pp. 292-301, Jan. 2013.

[4] S. U. Rehman; A. Shabra, "A Temperature Estimation Method Using the Ratio of Emitter-to-Base Voltages," in IEEE Transactions on Very Large Scale Integration (VLSI) Systems, vol.PP, no.99, pp.1-9.

[5] B.-S. Song and P. R. Gray, "A precision curvaturecompensated CMOS bandgap reference," IEEE J. of SolidState Circuits, vol. SC-18, no. 6, pp. 634-643, Dec. 1983.

[6] T.Oshita, J. Shor, D.E. Duarte, A. Kornfeld, D. Zilberman, "Compact BJT-Based Thermal Sensor for Processor Applications in a $14 \mathrm{~nm}$ tri-Gate CMOS Process," IEEE J. Solid-State Circuits, vol.50, no.3, pp.799-807, 2015.

[7] Wenfeng Zhao; Rui Pan; Yajun Ha; Zhi Yang, "A 0.4V 280$\mathrm{nW}$ frequency reference-less nearly all-digital hybrid domain temperature sensor," IEEE Asian Solid State Circuits Conference, pp.301-304, 10-12 Nov. 2014.

[8] J. Shor, K. Luria, D. Zilberman, "Ratiometric BJT-based thermal sensor in $32 \mathrm{~nm}$ and $22 \mathrm{~nm}$ technologies," IEEE ISSCC Dig. Tech. Papers pp.210-212, 2012 